\title{
Fetal alcohol exposure and development of the integument
}

This article was published in the following Dove Press journal:

Research and Reports in Neonatology

6 May 2016

Number of times this article has been viewed

\section{William D Longhurst Jordan Ernst ${ }^{2}$ \\ Larry Burd ${ }^{3}$}

'Center for Emergency Medicine, University Hospitals Case Medical Center, Case Western Reserve University, Cleveland, OH, USA; 2University of North Dakota School of Medicine and Health Sciences, Grand Forks, ND, USA; ${ }^{3}$ Department of Pediatrics, North Dakota Fetal Alcohol Syndrome Center, University of North Dakota School of Medicine and Health Sciences, Grand Forks, ND, USA
Correspondence: Larry Burd Department of Pediatrics, North Dakota Fetal Alcohol Syndrome Center, University of North Dakota School of Medicine and Health Sciences,

Grand Forks, ND 58202, USA

Tel + I 7017773683

Fax +I 7017774474

Email larry.burd@med.und.edu
Background: The physiology of fetal alcohol exposure changes across gestation. Early in pregnancy placental, fetal, and amniotic fluid concentrations of alcohol exposure are equivalent. Beginning in mid-pregnancy, the maturing fetal epidermis adds keratins which decrease permeability resulting in development of a barrier between fetal circulation and the amniotic fluid. Barrier function development is essential for viability in late pregnancy and in the extra-uterine environment. In this paper we provide a selected review of the effects of barrier function on fetal alcohol exposure.

Methods: We utilized a search of PubMed and Google for all years in all languages for MeSH on Demand terms: alcohol drinking, amnion, amniotic fluid, epidermis, ethanol, female, fetal development, fetus, humans, keratins, permeability, and pregnancy. We also reviewed the reference lists of relevant papers and hand-searched reference lists of textbooks for additional references.

Results: By 30 gestational weeks, development of barrier function alters the pathophysiology of ethanol dispersion between the fetus and amniotic fluid. Firstly, increases in the effectiveness of barrier function decreases the rate of diffusion of alcohol from fetal circulation across fetal skin into the amniotic fluid. This reduces the volume of alcohol entering the amniotic fluid. Secondly, barrier function increases the duration of fetal exposure by decreasing the rate of alcohol diffusion from amniotic fluid back into fetal circulation. Ethanol is then transported into maternal circulation for metabolism or elimination.

Conclusion: In late pregnancy, barrier function modifies alcohol diffusion rates across the epidermis back into fetal circulation. This increases the duration of exposure from each episode of drinking. This information may be useful for clinicians who care for women with alcohol use disorders during pregnancy, and may also be useful in explaining the rationale for avoiding alcohol use throughout pregnancy, including late pregnancy.

Keywords: fetal, exposure, ethanol, integument, fetal alcohol spectrum disorders, amniotic fluid, alcohol

\section{Introduction}

Antenatal alcohol exposure is a well-known risk factor for a wide range of adverse outcomes. ${ }^{1}$ In the United States, over half of non-pregnant women of child bearing age report alcohol use within the past month and the number of unintended pregnancies from 2001 to 2008 rose from $48 \%$ to $51 \%$ with little change in the overall pregnancy rate. ${ }^{2,3}$ It is estimated that prenatal alcohol exposure occurs in about $40 \%$ of pregnant women in the United States during early pregnancy with about 2\%-4\% $(80,000$ to 160,000) having exposure during all three trimesters. ${ }^{4}$

The teratogenic effects of prenatal alcohol exposure are associated with adverse effects to every organ system. ${ }^{5}$ The most well-known effects include alcohol-related 
birth defects, abnormal facial features, growth impairment, and neurodevelopmental disorders collectively referred to as fetal alcohol spectrum disorders (FASD). ${ }^{6,7}$ The endophenotype of FASD is highly variable. ${ }^{8}$ Thresholds of FASD expression and severity have multiple effect modifiers which include socio-economic status, nutrition, genetics, smoking, polysubstance abuse, dosimetry, timing and duration of exposure during pregnancy. ${ }^{4,9}$ Prevalence rates for FASD vary from one to 800 per $10,000 .{ }^{10-12}$ FASD remains the leading identifiable cause of intellectual disability and one of the most preventable developmental disorders. ${ }^{4}$

Human studies have identified diverging alcohol concentrations in the maternal blood, fetal blood, and amniotic fluid during mid to late pregnancy., ${ }^{9,13}$ In a sample of six healthy pregnant women at 16-18 weeks gestation, maximal ethanol concentrations were lower (two-fold) in the amniotic fluid compared to maternal levels. ${ }^{14,15}$ The alcohol elimination rate from amniotic fluid was about half the rate of maternal circulation. After 3.5 hours when alcohol was no longer detectable in maternal blood, it was still detectable in amniotic fluid. ${ }^{14,15}$ Furthermore, the ethanol area under the curve, 0-3.5 hours in amniotic fluid, was only $16 \%$ lower than the value for maternal blood. ${ }^{15}$ These concentration differences persist for hours, thereby suggesting that some barrier to the equilibration of alcohol concentrations must be present beginning in mid-pregnancy. ${ }^{13}$ These differences likely result from changes in the maturing fetal skin starting around the second trimester. In this paper, we review published literature to provide an updated summary of the effects of development of fetal skin on alcohol exposure during pregnancy.

\section{Methods}

We utilized an electronic search of PubMed and Google for all years in all languages for the following MeSH on Demand terms: alcohol drinking, amnion, amniotic fluid, epidermis, ethanol, female, fetal development, fetus, humans, keratins, permeability, and pregnancy. The search terms also included integument, skin, fetal, prenatal, barrier function, and gestation. We then reviewed the reference lists of relevant papers and hand-searched reference lists of textbooks to identify references on the topic of effects of fetal skin development on prenatal alcohol exposure.

\section{Results}

\section{Early pregnancy}

At 11 gestational weeks the surface area of the fetus is $30 \mathrm{~cm}^{2}$ and increases 70 -fold to $2,200 \mathrm{~cm}^{2}$ at term. ${ }^{16}$ Fetal skin is highly permeable which facilitates the formation of amniotic fluid as a transudate of fetal plasma. ${ }^{17}$ In the first weeks of gestation, the fetal skin begins as a single layer of highly permeable ectodermal cells. By 4 weeks gestation, these cells develop into a layered integument with a metabolically active basal layer and a permeable outer periderm which interfaces with the amniotic fluid. ${ }^{17,18}$ At the beginning of pregnancy, amniotic fluid volume exceeds fetal volume. By the 20th week of gestation the two volumes are equal, by the 30th week of gestation amniotic fluid volume is approximately half the fetal volume, and at term it is approximately a quarter of fetal volume. ${ }^{9}$ By 40 weeks gestation, approximately 4 liters of water are present within the human fetal compartment, with 2,800 mL in the fetus, $400 \mathrm{~mL}$ in the placenta, and $800 \mathrm{~mL}$ in the amniotic fluid. ${ }^{19}$ Amniotic fluid has a turnover rate of approximately once per day., ${ }^{9}$

Ethanol has a kinetic diameter of 4.46 angstroms which is about twice that of water (2.68 angstroms) making ethanol $100 \%$ miscible in water. ${ }^{20}$ Ethanol's volume of distribution is approximately 0.45 to $0.6 \mathrm{~L} / \mathrm{kg} .{ }^{15,21}$ Following maternal ingestion, alcohol readily diffuses across the fetal skin into the amniotic fluid. Alcohol concentrations in the fetal compartment rise and equilibrate to concentrations that are nearly equivalent to maternal concentrations within 1-2 hours. However, reduction in blood alcohol concentrations in the fetal compartment occurs at a much slower rate than in the maternal circulation. ${ }^{22}$

At its peak the fetal metabolic capacity for ethanol is only $5 \%-10 \%$ of the maternal capacity. Therefore, effective clearance of alcohol from the fetal compartment relies on transfer back into maternal circulation for elimination. Rates of diffusion of ethanol from the amniotic fluid into the fetal circulation and ultimately into the maternal circulation, determine the duration of the alcohol exposure episode. In the first half of pregnancy, the increased permeability of the epidermis and peridermal layer expedites alcohol diffusion into and elimination from the fetal compartment.

\section{Development of epidermal barrier function during mid to late pregnancy prolongs fetal exposure to alcohol}

The kinetics of alcohol dispersion and elimination are influenced by the form and function of the evolving epidermis. By 20 weeks the surface area of the fetal skin is about $500 \mathrm{~cm}^{2} .{ }^{16}$ Studies of post-mortem skin samples from separate anatomical sites of infants between 17 and 30 weeks gestational age demonstrated that barrier activity develops regionally. Barrier function begins at approximately 20-24 weeks of gestation and global barrier function occurs by gestational week $30 .^{23}$ 
By 18 weeks of gestation vernix caseosa begins to cover the fetal skin with a lipid rich product mixed with periderm cells that are secreted by the sebaceous glands. ${ }^{24-26}$ The vernix is comprised of fetal corneocytes with very high water content which also contributes to barrier function and limits diffusion of ethanol. ${ }^{23,26,27}$ Thus, transport of ethanol from the amniotic fluid into the fetal circulation may be the rate-limiting step for the elimination of ethanol from amniotic fluid. Before 20 weeks of gestation, this may not be a factor because reabsorption occurs through the fetal skin. After 20 weeks of gestation, transport occurs by means of two pathways: fetal swallowing and the intramembranous pathway (Figure 1). 17,28

Fetal swallowing begins during the 11th week of gestation. By term, the amount of amniotic fluid swallowed by the fetus is between 500 and $1,000 \mathrm{~mL} /$ day. ${ }^{29}$ The second route for reabsorption into the fetal circulation is the intermembranous pathway. This pathway involves the absorption of amniotic fluid across the amnion and into the fetal vasculature driven by osmotic differences. ${ }^{29}$

In the last two trimesters of pregnancy, fetal exposure to ethanol is further prolonged. This is the result of the recurrent cycle of fetal swallowing of the amniotic fluid containing ethanol, and elimination of urine containing alcohol back into the amniotic fluid. ${ }^{4,9}$

Another factor affecting the alcohol elimination rate from the fetal compartment is the effect of ethanol on umbilical vessels which react to ethanol at very low levels $(50-85 \mathrm{mg} / \mathrm{dL}) .^{30}$ The umbilical artery is even more sensitive to ethanol exposure, with $10 \%$ of arteries demonstrating vasoconstriction at exposure levels beginning at $0.001 \mathrm{~g}$ (exposure to approximately one standard drink). ${ }^{31}$ This effect may be further modified by the effects of alcohol-induced vascular maladaptations occurring at the maternal fetal interface. ${ }^{32}$

Morphologic changes observed in the epidermis correlated well with barrier development and include disaggregation of periderm coinciding with increasing keratinization and layer formation in the stratum corneum. ${ }^{23}$ Later in pregnancy the fetal epidermis develops an important role in the regulation of amniotic fluid volume and composition including exchange of water, sodium, and possibly glucose..$^{33}$ Newly acquired epidermal barrier activity has major implications on the composition and circulation of amniotic fluid. As barrier function increases fetal plasma dialysate no longer significantly contributes to amniotic fluid volume; rather the major contribution is from fetal urine and to a lesser extent pulmonary secretions. ${ }^{23}$ Fetal swallowing occurs as early as 8 weeks gestation, but does not have an appreciable effect on fluid circulation until development of barrier activity in the fetal skin. ${ }^{28}$ These developmental changes modify the route and rate of alcohol elimination.

By 20 weeks gestation, keratinization of the fetal skin results in the development of an epidermal barrier reducing permeability and inhibiting the bi-directional diffusion of water and other solutes between fetal circulation and amniotic fluid. ${ }^{33}$ Development of the barrier function during mid-to-late pregnancy impairs rapid diffusion of ethanol across the large surface area of fetal skin. These developmental changes result in decreased rates of alcohol elimination from the fetal compartment. The result is that, in the last half of pregnancy, amniotic fluid functions as a reservoir for alcohol. ${ }^{9}$ In late pregnancy alcohol is recirculated from amniotic fluid into fetal

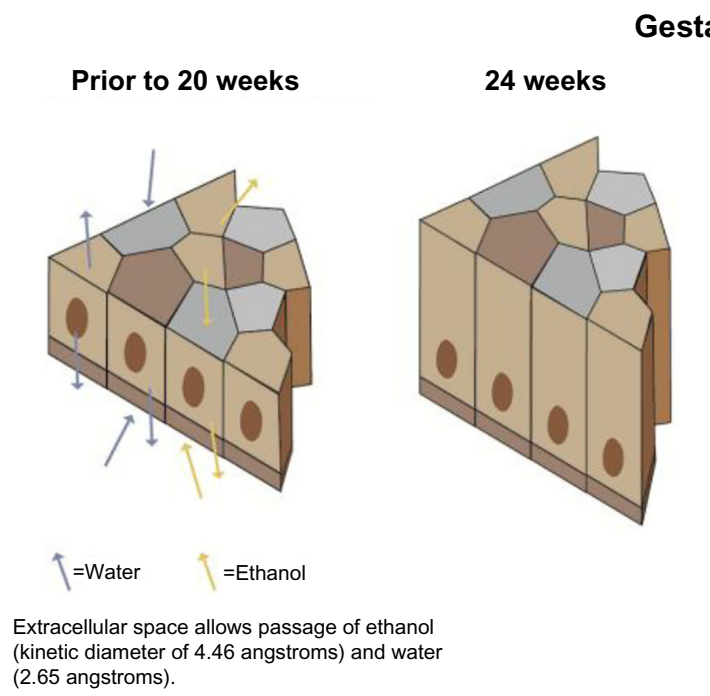

\section{estational age}

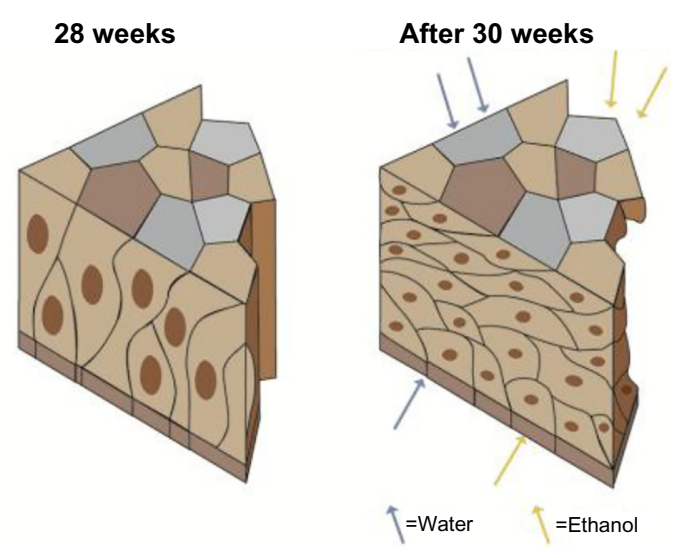

Development of barrier function reduces extracellular space to below 2.6 angstroms and prevents passive diffusion of ethanol and water

Figure I Graphic of fetal integument development and development of barrier function. 
circulation primarily by fetal swallowing. A small fraction is metabolized by fetal enzymes, some is re-excreted into amniotic fluid in fetal urine, and the majority is transferred into maternal circulation for elimination. The amount of amniotic fluid swallowed by the fetus (500-1,000 mL/day) does not account for the amount of urine and pulmonary secretions (970-1,370 $\mathrm{mL} /$ day) that enter the amniotic fluid. ${ }^{34}$ An alternate route of fluid absorption must account for the difference, with the intramembranous pathway having the most support in the literature. The pathway details the absorption of amniotic fluid across the amnion and into the fetal vasculature driven by osmotic differences. ${ }^{34}$

The absorption of the amniotic fluid through fetal swallowing and the intramembranous pathway creates a recycling system where much of the ethanol that the fetus excretes will be reabsorbed back into its circulatory system. ${ }^{22}$ Thus, the barrier function of the developing epidermis creates a cycle of repeated reintroduction of alcohol into the amniotic fluid which prolongs the duration of each exposure episode during late pregnancy. ${ }^{9}$

\section{Discussion}

The pathophysiology of prenatal alcohol exposure is an important public health issue since $50 \%$ of pregnancies are complicated by alcohol exposure and adverse outcomes from exposure affect at least $1 \%$ of live births. ${ }^{35}$ In response, numerous professional societies have developed warnings about the risks from drinking prior to and during pregnancy often emphasizing that no amount of alcohol is safe during pregnancy. ${ }^{36-39}$

A more complete understanding of the complex interactions between exposure and development provides clinicians with a physiological rationale for discouraging maternal alcohol use at any time during pregnancy. The effects of development of the fetal integument is an understudied but important component of the appreciation of risk to the fetus from alcohol exposure especially in late pregnancy. Maturation of fetal skin and the progressive development of barrier function modifies both the route and rate of alcohol elimination from the fetal compartment in late pregnancy. Consequently, alcohol clearance from the amniotic fluid is prolonged in late pregnancy, which lengthens the duration of fetal alcohol exposure from each episode of maternal alcohol ingestion.

\section{Clinical implications}

Multiple adverse outcomes from prenatal alcohol exposure are manifest in late pregnancy and at delivery these include increased risk of stillbirth, extreme prematurity, prematurity, and infectious illness. ${ }^{40}$ Paradoxically, premature delivery acts as an effect modifier and reduces late pregnancy exposure and the cumulative exposure during pregnancy. These changes also occur in the context of changes from ethanol exposure. As an example, ethanol may alter fetal kidney function by either vasoconstriction of the vasculature or from abnormalities in kidney structure. This may affect the production of fetal urine which is the primary constituent of amniotic fluid.

Since many infants with fetal alcohol exposure are unidentified, risks associated with third trimester exposure are likely underappreciated. ${ }^{40-42}$ Inability to confirm alcohol exposure during pregnancy is a frequent problem in the diagnosis of FASD later in life. Improved screening and increased clinical suspicion for fetal alcohol exposure may improve rates of identification at delivery and in the neonatal intensive care nursery. Hospital stays for infants with fetal alcohol syndrome are increased by three-fold (12.1 days vs 3.9 days for matched controls). ${ }^{40,43}$ Thus, an adequate time window for more detailed assessment of exposure is available. This also allows time to consider interventions to reduce likelihood of exposure in subsequent pregnancies.

\section{Future research}

Several important research activities are needed to further improve our understanding of fetal alcohol exposure.

1. Future research should include study of routine exposure assessments for all children entering neonatal intensive care units. In addition to estimating prevalence, the projects should include attention to epidemiological endpoints (accuracy, sensitivity, specificity, cost per infant, and cost per case identified). This will facilitate comparison with other studies of exposure assessment strategies to facilitate the identification of optimal detection methodologies.

2. Increasing the training and sophistication of maternal interviewers is a key strategy to improve exposure detection. Future research should evaluate both the cost and benefits of utilizing specially trained staff for this complex task. Consideration must be given to the development of a clinical specialization for both exposure assessment and management of alcohol and other substance exposed pregnancies. This should include physicians, nurses, and midwives.

3. Another important key is to increase levels of clinical suspicion of prenatal alcohol use. In the United 
States $50 \%$ of newborns will have had at least minimal alcohol exposure during pregnancy. At least 4\% will have exposure across all three trimesters, and very few are identified. Amazingly, many clinicians believe that alcohol use is common but they often feel that it is uncommon in the patient population they care for. Due in part to poor or missing exposure information, over $90 \%$ of cases of FASD are undiagnosed. A recent study from Australia found that only $45 \%$ of health care providers ask about alcohol use during pregnancy, and only $25 \%$ routinely provide their patient with information about the effects of alcohol on the fetus. ${ }^{44}$ Thus, improvements are needed to identify and appropriately care for these children and their mothers, and to prevent exposure during subsequent pregnancies.

4. A key research strategy will be the development of exposure detection panels for use by clinicians rather than relying on single test strategies. Figure 2 is a potential example. This strategy should include ongoing study of strategies for prenatal alcohol detection that are deployed for routine use across prenatal care settings. ${ }^{35,45}$ Where possible these tools should be brief and built into the electronic medical record.

5. The exposure detection panels should also include studies of markers for both alcohol exposure and smoking since the interactions are not additive, but multiplicative. ${ }^{46}$

6. Increased emphasis should be placed on identification of biomarkers of exposure in amniotic fluid. Alcohol may be present in amniotic fluid after it is undetectable in maternal blood. In nearly all cases women who are drinking in late pregnancy will have been drinking throughout pregnancy resulting in the highest levels of cumulative exposure. As a result, research in late pregnancy offers a very important time period for the identification of diverse biomarkers of exposure. These should be linked to both acute and chronic exposure. Lastly, biomarkers of growth impairment and neurocognitive impairment are urgently needed.

7. Additional research should also focus on identification of biomarkers for mortality risk. It would be very helpful to further examine levels of alcohol required to suppress fetal breathing movements and to determine if very low levels of alcohol in amniotic fluid may continue to affect respiratory effort at delivery.

While rates of other substance abuse have a waxing and waning course, rates of prenatal alcohol exposure remain at alarmingly high levels. Unfortunately, epidemics of abuse of cocaine, crack cocaine, methamphetamine, heroin use, and most recently increased rates of prescription pain medications, especially the opioids, have overshadowed concern about alcohol exposure. The prevalence rates of these substances rarely match the prevalence of prenatal alcohol exposure which has persisted, largely unchanged for decades. This is a worldwide problem especially in the developing world, with high rates in Africa. ${ }^{35}$ Prenatal alcohol exposure, and its sequela of stillbirth, prematurity, infant mortality, and the lifelong neuropsychiatric impairments must not be overlooked in pregnancies complicated by other substance abuse. In these pregnancies, alcohol use is very likely present and may be the underlying teratogen accounting for a considerable proportion of the phenotype. Lastly, this issue deserves attention for the sake of the next infant born to these mothers where exposure may be prevented, which is the ultimate in prevention strategies.

The protocol in Figure 2 would ideally be routinely administered by placing it in the electronic medical record. The multi-element process begins with the One Question Screen "When was your last drink?" If any alcohol use during pregnancy is reported, the data of the last drink are used to risk stratify alcohol use. No drinking during pregnancy is stratified as unexposed. Alcohol use before recognition of pregnancy but no use since recognition of pregnancy is classified as exposed. Alcohol use after recognition of pregnancy is classified as exposed and high risk and immediate intervention is recommended.

The last drink question can also be very helpful in dating epochs of exposure. When drinking is reported, estimates of frequency and dosimetry are charted and used to estimate cumulative exposure for the pregnancy. These are straightforward calculations and are important to chart. Importantly, the data are estimated with the definition of what a drink is, so more specific estimates of exposure can be estimated using drink volume, frequency of drinking, and alcohol content of a drink. If no use is reported, but clinical concerns about exposure continue, a maternal risk score can be calculated. Scores above 30 suggest exposure is likely and that the level of exposure may place the fetus at risk for an FASD. If concerns still exist, biomarker studies can also be considered if either hair or meconium is available. Meconium analysis can detect exposure over the last 20 weeks and especially over the last trimester of pregnancy. ${ }^{47}$ Maternal or infant hair may also be used.

In exposure assessment, the importance of training interviewers cannot be overemphasized. Detection of exposure in 


\section{Fetal alcohol exposure assessment}

\section{Exposure risk markers}

- History of maternal substance abuse

- Premature birth (<36 weeks)

Congenital defects

- Family expresses concerns about substance abuse

- Emergency room visit with concerns about substance abuse

- Mother smokes

- Mother has been in jail or prison

Screening for prenatal alcohol exposure

\begin{tabular}{|c|c|c|}
\hline \multicolumn{3}{|c|}{ When was your last drink? } \\
\hline$\downarrow$ & $\downarrow$ & $\downarrow$ \\
\hline Before & \multicolumn{2}{|c|}{ During pregnancy } \\
\hline & Pre-awareness & Post-awareness \\
\hline Unexposed & Exposed & $\begin{array}{l}\text { Exposed and } \\
\text { high risk }\end{array}$ \\
\hline \multicolumn{3}{|c|}{ Risk stratification } \\
\hline Unexposed & Exposed & $\begin{array}{l}\text { Exposed and } \\
\text { high risk }\end{array}$ \\
\hline
\end{tabular}

No use reported, but concerns remain

\section{Maternal risk score}

Maternal risk score
Age over 25 years
Unmarried, divorced, widow, living with partner
On TANF, WIC, social security or
income < $\$ 16,000$ per year
Did not graduate from high school

\section{Assessment of exposure during pregnancy}

On average, how many days per week
did you drink during pregnancy?
On an average drinking day during pregnancy,
how many drinks did you have?
How many days per month did you have
4 or more drinks during pregnancy?
What is the most you had to drink
on any one day during pregnancy?
What is a drink? Alcohol\%

\section{Calculate exposure parameters}

(Cumulative exposure during pregnancy)

Pregnancy drinking days $=(a \times 40)=$

Estimates number of drinking days during pregnancy.

Percentage of days exposed

during pregnancy $=(e+280)=$

Estimates days exposed during pregnancy.

Number of binge days
(4 or more drinks in one

(4 or more drinks in one day)

Estimates number of binge days.

Number of drinks

during pregnancy $\quad=(a \times b \times 40)=$

Estimates cumulative number of drinks during pregnancy.

Ounces of absolute alcohol $=(f+2)=$

Estimates cumulative absolute alcohol exposure during pregnancy.

If concerns remain, order:

Meconinum or hair analysis

$\diamond$ Fatty acid ethyl esters

- Ethyl glucuronide

Figure 2 A model of an exposure assessment.

Abbreviations: TANF, temporary assistance for needy children; WIC, women, infants and children; FASD, fetal alcohol spectrum disorders; vol, volume.

our experience is often comprised by use of minimally trained interviewers. Interviewers need training with experienced mentors to become skilled interviewers.

Prenatal alcohol exposure is both common and complex. We have much to learn about the teratogenic effects of alcohol during pregnancy and the interplay of exposure and embryology of late pregnancy.

\section{Disclosure}

The authors have no conflicts of interest to disclose.

\section{References}

1. US Department of Health and Human Services. U.S. Surgeon General Releases Advisory on Alcohol Use in Pregnancy [press release). US Department of Health and Human Services; 2005. Available from: http:// come-over.to/FAS/SurGenAdvisory.htm. Accessed February 6, 2016. 
2. Centers for Disease Control and Prevention (CDC). Alcohol use and binge drinking among women of childbearing age - United States, 2006-2010. MMWR Morb Mortal Wkly Rep. 2012;61(28): 534-538.

3. Finer LB, Zolna MR. Shifts in intended and unintended pregnancies in the United States, 2001-2008. Am J Public Health. 2014;104 (Suppl 1): S43-S48.

4. Paintner A, Williams AD, Burd L. Fetal alcohol spectrum disorders implications for child neurology, part 1: prenatal exposure and dosimetry. J Child Neurol. 2012;27(2):258-263.

5. Popova S, Lange S, Shield K, et al. Comorbidity of fetal alcohol spectrum disorder: a systematic review and meta-analysis. The Lancet. 2016;387(3):978-987.

6. Pruett D, Waterman EH, Caughey AB. Fetal alcohol exposure: consequences, diagnosis, and treatment. Obstet Gynecol Surv. 2013;68(1):62-69.

7. Blum A, Loser H, Dehaene P, Rassner G. Fetal alcohol syndrome (FAS) in dermatology: an overview and an evaluation. Eur J Dermatol. 1999;9(5):341-345.

8. Burd L, Klug MG, Martsolf J, Kerbeshian J. Fetal alcohol syndrome: neuropsychiatric phenomics. Neurotoxicol Teratol. 2003;25(6):697-705.

9. Heller M, Burd L. Review of ethanol dispersion, distribution, and elimination from the fetal compartment. Birth Defects Res A Clin Mol Teratol. 2014;100(4):277-283.

10. Hymbaugh K, Miller LA, Druschel CM, et al. A multiple source methodology for the surveillance of fetal alcohol syndrome - The Fetal Alcohol Syndrome Surveillance Network (FASSNet). Teratology. 2002;66 (Suppl 1):S41-S49.

11. May PA, Gossage JP, Kalberg WO, et al. Prevalence and epidemiologic characteristics of FASD from various research methods with an emphasis on recent in-school studies. Dev Disabil Res Rev. 2009;15(3):176-192.

12. May PA, Baete A, Russo J, et al. Prevalence and characteristics of fetal alcohol spectrum disorders. Pediatrics. 2014;134(5):855-866.

13. Idanpaan-Heikkila J, Jouppila P, Akerblom HK, Isoaho R, Kauppila E, Koivisto M. Elimination and metabolic effects of ethanol in mother, fetus, and newborn infant. Am J Obstet Gynecol. 1972;112(3): 387-393.

14. Brien JF, Loomis CW, Tranmer J, McGrath M. Disposition of ethanol in human maternal venous blood and amniotic fluid. Am J Obstet Gynecol. 1983;146(2):181-186.

15. Zelner I, Koren G. Pharmacokinetics of ethanol in the maternal-fetal unit. J Popul Ther Clin Pharmacol. 2013;20(3):e259-e265.

16. Meban C. The surface area and volume of the human fetus. $J$ Anat. 1983;137(Pt 2):271-278.

17. Beall MH, van den Wijngaard JP, van Gemert MJ, Ross MG. Amniotic fluid water dynamics. Placenta. 2007;28(8-9):816-823.

18. Hoath SB, Maibach HI, editors. Neonatal skin structure and function. New York: Marcel Dekker, Inc.; 2003.

19. Modena AB, Fieni S. Amniotic fluid dynamics. Acta Biomed. 2004;75 (Suppl 1):11-13.

20. Borjigin T, Sun F, Zhang J, Cai K, Ren H, Zhu G. A microporous metalorganic framework with high stability for GC separation of alcohols from water. Chem Commun (Camb). 2012;48(61):7613-7615.

21. Kalant H, Khanna J. The alcohols. In: Kalant H, Grant D, Mitchell J, editors. Principles of medical pharmacology. 7 th ed. Toronto, ON Canada: Elsevier; 2007:275-288.

22. Burd L, Blair J, Dropps K. Prenatal alcohol exposure, blood alcohol concentrations and alcohol elimination rates for the mother, fetus and newborn. $J$ Perinatol. 2012;32(9):652-659.

23. Hardman MJ, Moore L, Ferguson MW, Byrne C. Barrier formation in the human fetus is patterned. J Invest Dermatol. 1999;113(6): 1106-1113.

24. Hoath SB, Narendran V, Visscher MO. The biology and role of vernix. Newborn and Infant Nursing Reviews. 2001;1(1):53-58.

25. Pocki P. The sebaceous gland. In: Maibach H, Berardesca E, editors. Neonatal skin. New York, NY: Marcel Dekker; 1982:67-80.
26. Singh G, Archana G. Unraveling the mystery of vernix caseosa. Indian $J$ Dermatol. 2008;53(2):54-60.

27. Pickens WL, Warner RR, Boissy YL, Boissy RE, Hoath SB. Characterization of vernix caseosa: water content, morphology, and elemental analysis. J Invest Dermatol. 2000;115(5):875-881.

28. Underwood MA, Gilbert WM, Sherman MP. Amniotic fluid: not just fetal urine anymore. $J$ Perinatol. 2005;25(5):341-348.

29. Gilbert WM, Brace RA. The missing link in amniotic fluid volume regulation: intramembranous absorption. Obstet Gynecol. 1989; 74(5):748-754.

30. Altura BM, Altura BT, Carella A, Chatterjee M, Halevy S, Tejani N. Alcohol produces spasms of human umbilical blood vessels: relationship to fetal alcohol syndrome (FAS). Eur J Pharmacol. 1982;86(2):311-312.

31. Savoy-Moore RT, Dombrowski MP, Cheng A, Abel EA, Sokol RJ. Low dose alcohol contracts the human umbilical artery in vitro. Alcohol Clin Exp Res. 1989;13(1):40-42.

32. Ramadoss J, Magness RR. Vascular effects of maternal alcohol consumption. Am J Physiol Heart Circ Physiol. 2012;303(4):H414-H421.

33. Holbrook KA, Odland GF. The fine structure of developing human epidermis: light, scanning, and transmission electron microscopy of the periderm. J Invest Dermatol. 1975;65(1):16-38.

34. Gilbert WM. The cost of preterm birth: the low cost versus high value of tocolysis. BJOG. 2006;113 (Suppl 3):4-9.

35. Williams AD, Nkombo Y, Nkodia G, Leonardson G, Burd L. Prenatal alcohol exposure in the Republic of the Congo: prevalence and screening strategies. Birth Defects Res A Clin Mol Teratol. 2013;97(7):489-496.

36. No authors listed. American Academy of Pediatrics. Committee on Substance Abuse and Committee on Children With Disabilities. Fetal alcohol syndrome and alcohol-related neurodevelopmental disorders. Pediatrics. 2000;106(2 Pt 1):358-361.

37. American College of Obstetricians and Gynecologists. Committee on Health Care for Underserved Women. Committee opinion no. 496: At-risk drinking and alcohol dependence: obstetric and gynecologic implications. Obstet Gynecol. 2011;118(2 Pt 1):383-388.

38. Moyer VA; Preventive Services Task Force. Screening and behavioral counseling interventions in primary care to reduce alcohol misuse: U.S. preventive services task force recommendation statement. Ann Intern Med. 2013;159(3):210-218.

39. Office of the Surgeon General. Press release. U.S. Surgeon General's Advisory on Alcohol Use in Pregnancy [press release]. Office of the Surgeon General; 2005. Available from: http://www.cdc.gov/mmwr/ preview/mmwrhtml/mm5409a6.htm. Accessed February 6, 2016.

40. Gauthier TW. Prenatal Alcohol Exposure and the Developing Immune System. Alcohol Res. 2015;37(2):279-285.

41. Little BB, Snell LM, Rosenfeld CR, Gilstrap LC 3rd, Gant NF. Failure to recognize fetal alcohol syndrome in newborn infants. Am J Dis Child. 1990;144(10):1142-1146

42. Stoler JM, Holmes LB. Under-recognition of prenatal alcohol effects in infants of known alcohol abusing women. $J$ Pediatr. 1999;135(4):430-436.

43. Kvigne VL, Leonardson GR, Borzelleca J, Neff-Smith M, Welty TK. Hospitalizations of children who have fetal alcohol syndrome or incomplete fetal alcohol syndrome. S D Med. 2009;62(3):97, 99, 101-3.

44. Payne J, Elliott E, D'Antoine H, et al. Health professionals' knowledge, practice and opinions about fetal alcohol syndrome and alcohol consumption in pregnancy. Aust N Z J Public Health. 2005;29(6):558-564.

45. Himes SK, Dukes KA, Tripp T, et al. Clinical sensitivity and specificity of meconium fatty acid ethyl ester, ethyl glucuronide, and ethyl sulfate for detecting maternal drinking during pregnancy. Clin Chem. 2015;61(3):523-532.

46. Odendaal HJ, Steyn DW, Elliott A, Burd L. Combined effects of cigarette smoking and alcohol consumption on perinatal outcome. Gynecol Obstet Invest. 2009;67(1):1-8.

47. Hofer R, Burd L. Review of published studies of kidney, liver, and gastrointestinal birth defects in fetal alcohol spectrum disorders. Birth Defects Res A Clin Mol Teratol. 2009;85(3):179-183. 
Research and Reports in Neonatology

Dovepress

\section{Publish your work in this journal}

Research and Reports in Neonatology is an international, peer-reviewed, open access journal publishing original research, reports, editorials, reviews and commentaries on neonatal health. The manuscript management system is completely online and includes a very quick and fair

peer-review system. Visit http://www.dovepress.com/testimonials.php to read real quotes from published authors. 